\title{
Acoustic rat-race coupler and its applications in non-reciprocal systems
}

Farzad Zangeneh-Nejad, and Romain Fleury

Citation: The Journal of the Acoustical Society of America 146, 843 (2019); doi: 10.1121/1.5115020

View online: https://doi.org/10.1121/1.5115020

View Table of Contents: https://asa.scitation.org/toc/jas/146/1

Published by the Acoustical Society of America

\section{ARTICLES YOU MAY BE INTERESTED IN}

Nonreciprocal acoustic propagation and leaky-wave radiation in a waveguide with flow

The Journal of the Acoustical Society of America 146, 802 (2019); https://doi.org/10.1121/1.5115018

Introduction to the special issue on non-reciprocal and topological wave phenomena in acoustics

The Journal of the Acoustical Society of America 146, 719 (2019); https://doi.org/10.1121/1.5119133

Non-reciprocal wave propagation in mechanically-modulated continuous elastic metamaterials

The Journal of the Acoustical Society of America 146, 782 (2019); https://doi.org/10.1121/1.5115019

Asymmetric acoustic energy transport in non-Hermitian metamaterials

The Journal of the Acoustical Society of America 146, 863 (2019); https://doi.org/10.1121/1.5114919

Reconfigurable topological insulator for elastic waves

The Journal of the Acoustical Society of America 146, 773 (2019); https://doi.org/10.1121/1.5114920

Asymmetric scattering of flexural waves in a parity-time symmetric metamaterial beam

The Journal of the Acoustical Society of America 146, 850 (2019); https://doi.org/10.1121/1.5116561

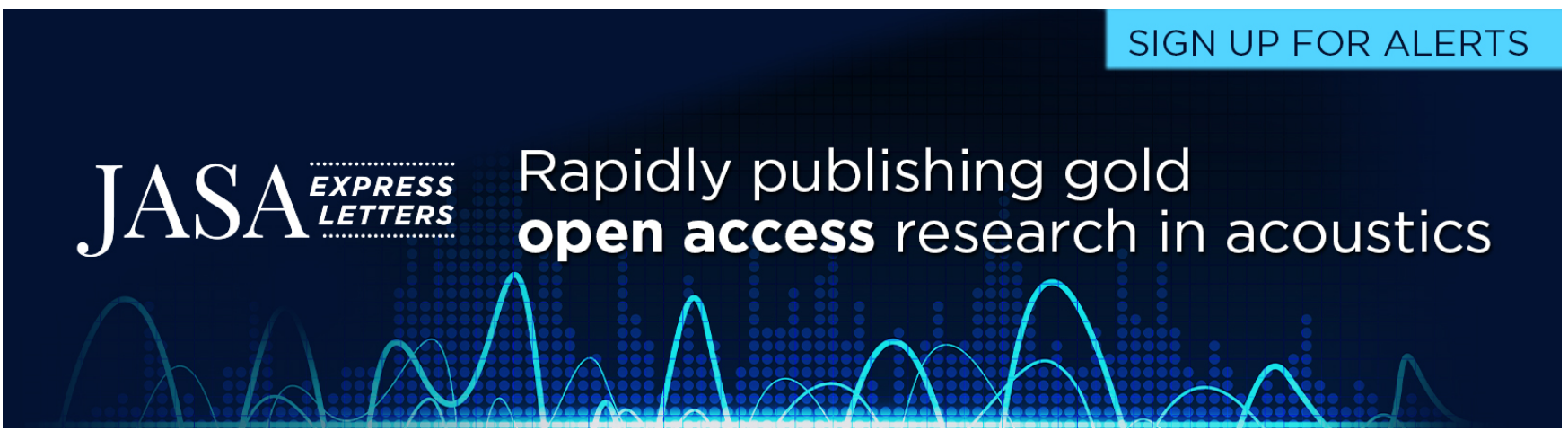




\title{
Acoustic rat-race coupler and its applications in non-reciprocal systems
}

\author{
Farzad Zangeneh-Nejad and Romain Fleury ${ }^{\text {a) }}$ \\ Institute of Electrical Engineering, EPFL, 1015 Lausanne, Switzerland
}

(Received 16 January 2019; revised 4 April 2019; accepted 9 April 2019; published online 31 July 2019)

Waveguide hybrid junctions, such as Magic-T and rat-race couplers, have been of great interest in microwave technology not only for their applications in power monitoring, but also for design and synthesis of various non-reciprocal devices including electromagnetic circulators and isolators. Here, an acoustic rat-race coupler is designed and demonstrated for the first time, working on the basis of constructive and destructive interferences between the clockwise and counterclockwise of a ring resonator. It is then shown how the sound isolation provided by such a coupler enables the realization of an acoustic four-port circulator, a device which has not been reported as yet. Many other promising acoustic devices comprising power combiners, power dividers, mixers, and modulators can be envisioned to be implemented based on the proposed rat-race coupler. (C) 2019 Acoustical Society of America. https://doi.org/10.1121/1.5115020

[JFL]

Pages: 843-849

\section{INTRODUCTION}

In the middle of 20th century, a large variety of microwave directional couplers were proposed including Bethe hole couplers, ${ }^{1}$ multi-hole waveguide couplers, ${ }^{2}$ T-junction waveguides, ${ }^{3}$ Wilkinson power dividers, ${ }^{4}$ waveguide magictees, and rat-race couplers. ${ }^{5}$ Such couplers, being capable of transmitting power over desired directions, then drew a considerable amount of attention for synthesizing a large variety of microwave components like power dividers, ${ }^{6}$ power combiners, ${ }^{7}$ and phase shifters, ${ }^{8}$ which are now widely used especially in power monitoring applications.

Among the above-mentioned types of couplers, the socalled magic-tee (or simply magic-T) hybrid junction and its alternative, the rat-race coupler, are probably the most important ones. ${ }^{9}$ The working principle of these devices, conceptually sketched in Figs. 1(a) and 1(b), is as follows: when power is fed into port 1 , it splits, equally in phase and amplitude, between ports 2 and 3 , while port 4 remains "magically" isolated from the excitation. On the other hand, the power injected into port 4 distributes, equally in amplitude but opposite in phase between ports 2 and 3 , yet without coupling to port $1 .^{9}$ Such magic isolation has made these junctions of large importance not only for power monitoring, but also for the development of various reciprocal and nonreciprocal devices like modulators, ${ }^{10}$ four-port circulators, ${ }^{11}$ duplexers, ${ }^{12}$ and mixers, ${ }^{13}$ to name a few.

Despite the wide-spread promotion of magic-T junctions in microwave engineering, there exists as yet no report on a similar junction or component offering a similar functionality for sound or other types of scalar waves. The reason might stem from the fact that, as more widely discussed in the following, magic- $\mathrm{T}$ hybrid junctions are polarizationbased, while scalar waves are longitudinal and do not possess a polarization degree of freedom. Consequently, when

\footnotetext{
${ }^{\text {a)} E l e c t r o n i c ~ m a i l: ~ r o m a i n . f l e u r y @ e p f l . c h ~}$
}

one forms a junction similar to Fig. 1(a) and injects sound, for example, into one port, the power always distributes among all other ports without leaving any of them isolated. Instead, resonant devices such as rat-race couplers, which do not work based on polarization, may be straightforwardly transferred from microwave to acoustics to achieve the same isolation functionality for sound waves.

In this article, we demonstrate, both theoretically and experimentally, an acoustic rat race coupler. The coupler works on the basis of constructive and destructive interferences between clockwise and counterclockwise acoustic modes of a ring resonator. We describe how the sound isolation provided by such a coupler enables the realization of an acoustic fourport circulator, a device which has not been reported so far. Just like their electromagnetic analogues, such couplers provide a roadmap for the synthesis of a large variety of reciprocal and non-reciprocal devices including power dividers, power combiners, mixers, duplexers, isolators, and modulators.

\section{SCATTERING MATRIX OF MAGIC-TEE HYBRIDS}

We start by briefly describing the scattering matrix of the magic-T hybrid junction shown in Fig. 1(a). In waveguide 1 , the electric field of the fundamental $T E_{10}$ mode is polarized along $y$ axis. Waveguide 4, however, is reversely polarized in the sense that its electric field is polarized along $x$. Assuming waveguide 1 is excited in its fundamental mode, the power is then equally split between waveguides 2 and 3 due to the symmetry of the structure, while waveguide 4 remains decoupled. By following the same arguments when the junction is excited from the other ports, one can write the scattering matrix of an ideal magic-T as ${ }^{9}$

$$
S=\frac{1}{\sqrt{2}}\left[\begin{array}{cccc}
0 & 1 & 1 & 0 \\
1 & 0 & 0 & -1 \\
1 & 0 & 0 & 1 \\
0 & -1 & 1 & 0
\end{array}\right]
$$


(a)

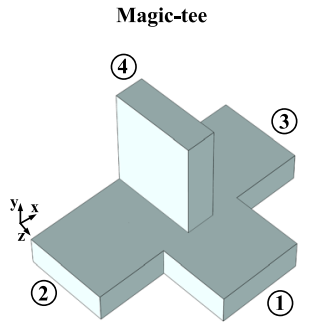

(b)

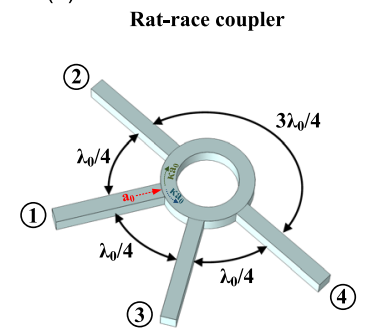

FIG. 1. (Color online) Magic tee hybrid junction. (a) A typical magic-T junction in microwave engineering, consisting of four arms crossing each other at a single junction. The underlying working principle of the device is based on the fact that the fundamental $T E_{10}$ modes have orthogonal polarization in arms 1 and 4. (b) A typical rat-race coupler mimicking exactly the behavior of the magic-T. The structure does not rely on polarization effects but works on the basis of constructive and destructive interferences between the clockwise and counterclockwise modes in a ring resonator.

It is instructive to underline that, according the obtained scattering matrix, when ports 2 and 3 are simultaneously excited, the sum of these two input fields appears at port 1 , while the received signal at port 4 is their difference. In this regard, ports 1 and 4 are sometimes known as sum and difference ports, respectively, while the two other ports are referred to as collinear arms in the literature.

Since the relatively broadband isolation of a microwave magic- $T$ is rooted into the different polarization of $T E_{10}$ modes inside the sum and difference arms, the junction does not provide isolation anymore if used for a wave of scalar nature such as airborne sound. In this case, an alternative with narrower bandwidth, namely a rat-race coupler, can be designed to provide a similar scattering matrix, as we demonstrate below.

\section{ACOUSTIC RAT-RACE COUPLER}

The geometry of the proposed rat-race coupler is represented in Fig. 1(b). It consists of four ports placed around one half of a ring resonator at $0,60,120$, and $180 \mathrm{deg}$. The distance between the ports at the frequency of optimal operation is $\lambda_{0} / 4$, while the total ring circumference is $3 \lambda_{0} / 2$. To gain some intuitive insight into the working principle of the structure, let us assume that an incident sound wave (with amplitude of $a_{0}$ ) is fed into port 1 . The injected wave can couple, with a coupling coefficient of $\kappa$ for instance, to the clockwise and counterclockwise modes of the ring. These clockwise and counterclockwise waves excite outgoing signals at ports 2 and 3 with the same phase, while their interference is destructive at port 4 . Therefore, their superposition gives rise to a zero pressure level at port 4 , and a pressure phasor of $-2 j \kappa a_{0}$ at ports 2 and 3. Likewise, when the device is excited from port 2 , the clockwise and counterclockwise modes interfere with each other destructively at port 3 while their constructive interference leads to the pressure phasors of $-2 j \kappa a_{0}$ and $2 j \kappa a_{0}$ at ports 1 and 4 , respectively. Following the same line of thoughts for the other two ports, one can obtain the scattering matrix of the four-port device as

$$
S=-2 j \kappa\left[\begin{array}{cccc}
0 & 1 & 1 & 0 \\
1 & 0 & 0 & -1 \\
1 & 0 & 0 & 1 \\
0 & -1 & 1 & 0
\end{array}\right]
$$

Notice that, in our analysis, we have assumed that the sound injected into the ports does not reflect back when reaching the junction. This assumption holds true whenever the characteristic impedance of the ring is $\sqrt{2}$ times that of the arms (see Appendix A). It should be further underlined that the unitary condition for the scattering matrix $S$ implies $\kappa=1 / 2 \sqrt{2}$. Hence, the scattering matrix $S$ reduces to

$$
S=-\frac{j}{\sqrt{2}}\left[\begin{array}{cccc}
0 & 1 & 1 & 0 \\
1 & 0 & 0 & -1 \\
1 & 0 & 0 & 1 \\
0 & -1 & 1 & 0
\end{array}\right]
$$

The obtained scattering matrix is exactly identical to that of the microwave magic tee: while all ports are connected to each other through the ring, one of them is always "magically" isolated from the others. Here, we have focused on an intuitive description of the scattering process, however a rigorous analysis based on the so-called even-odd mode analysis technique ${ }^{9}$ independently leads to Eq. (3) (see Appendix A).

In order to examine the proper performance of the proposed device, we performed full-wave finite-element numerical simulations of a realistic junction, assuming that it is excited from port 1 with a time-harmonic plane wave of unit amplitude at the wavelength $\lambda_{0}=15.3 \mathrm{~cm}$, which corresponds to the resonance frequency of the ring (see Appendix B for numerical methods and Appendix $\mathrm{C}$ for geometrical details). The acoustic pressure distribution (snapshot in time) is shown in Fig. 2(a). As expected, the input power couples out to ports 2 and 3, whereas the outgoing signal at port 4 is zero. Notably, the transmitted fields to waveguides 2 and 3 are out of phase with respect to the input signal, consistent with the scattering matrix of Eq. (3). The incident power is divided equally between ports 2 and 3. For further assertion, we calculate the frequency spectrum of the magnitude of the scattering parameters and plot them in Fig. 2(b). As seen in the figure, $S_{21}$ and $S_{31}$ become exactly equal at $f_{0}=c / \lambda_{0}$, whereas $S_{41}$ goes to zero at this frequency. In order to fully prove the proper functioning of the device, we further investigate the cases of excitation at other ports. Figures 2(c)-2(e) report the corresponding pressure field distributions when ports 2, 3, and 4 are excited, respectively. The obtained field patterns are in perfect agreement with the scattering matrix derived for the system.

We have built a prototype of such a coupler and evaluated its functionality using the experimental setup shown in Fig. 3(a). To probe the scattering behavior of the fabricated structure, we first excite the rat-race from port 2 with a loudspeaker driven by a burst noise voltage, and measure the amount of pressure at ports 1, 3, and 4. Insets of Fig. 3(b) depict the magnitude (left) and phase (right) of the resulting transfer functions versus frequency. It is clear that, near the resonance frequency of the rat-race coupler $f_{0}$, the 
(a)

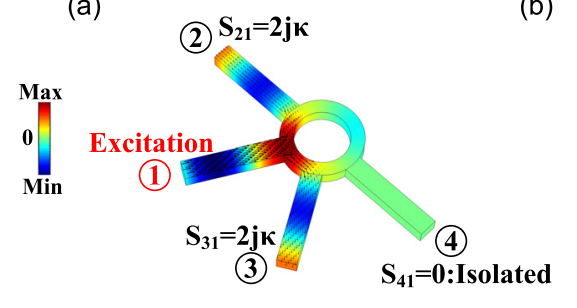

(c)

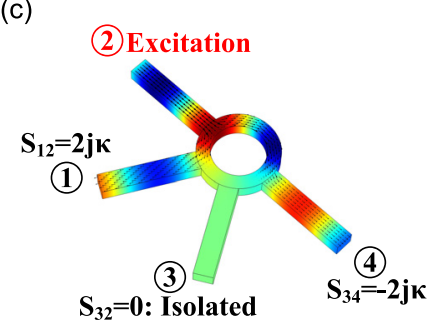

(d)

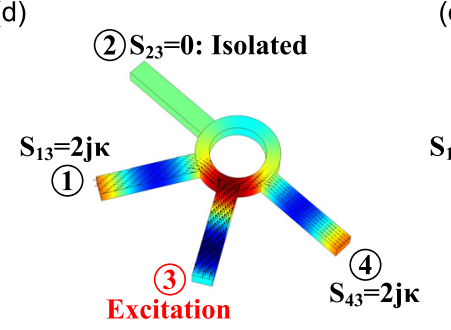

(b)

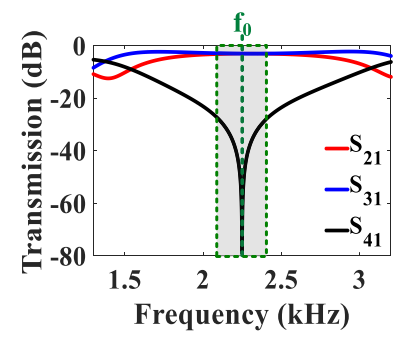

(e)

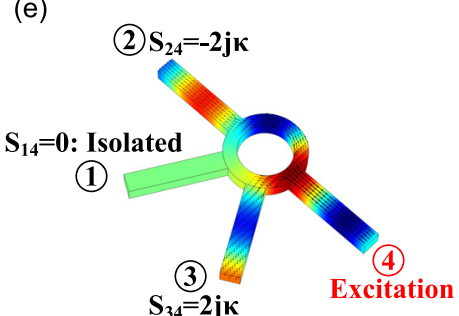

FIG. 2. (Color online) Demonstration of the proposed acoustic rat-race coupler. (a) An input sound wave is injected into port 1 and the corresponding pressure profile is calculated using full-wave numerical simulations. The wave is transmitted, with same phase and amplitude, to ports 2 and 3 while port 4 is isolated. (b) Variation of the scattering parameters versus frequency. The scattering parameters $S_{21}$ and $S_{31}$ become exactly equal at the frequency of desire $f_{0}$, whereas $S_{41}$ drops to zero at this frequency. (c)-(e) Same as panel (a) except that the structure is excited from ports $2,3,4$, respectively.

parameters $H_{42}$ and $H_{12}$ become identical in magnitude but are opposite in phase. One further notices that, as expected, $H_{32}$ approaches zero at this frequency. We next put the loudspeaker at port 1 and measure the magnitude [Fig. 3(c), left] and phase [Fig. 3(c), right] of the corresponding transfer functions $H_{i 1}(i=2,3,4)$. It is obvious that $H_{21}$ and $H_{31}$ have the same magnitude and phase over a relatively large frequency range around $f_{0}$ (grey area), whereas $H_{41}$ is close to zero within this frequency range. Our experimental results are consistent with our prior numerical findings.

\section{ACOUSTIC FOUR-PORT CIRCULATOR}

Not only may the sound isolation of the proposed acoustic rat-race coupler be of importance for power monitoring applications, but it also establishes an interesting platform for designing a new class of acoustic devices whose realization have not been possible before. An important example could be a four-port circulator. As schematically shown in Fig. 4(a), a four-port circulator is a non-reciprocal device where the power fed into each port is solely transmitted to the next port in rotation. Such circulators have already been realized for microwaves, yet not in acoustics, using two magic-tees and a gyrator, and have found important applications in radar systems and networks. ${ }^{11}$ We already realized the magic-tees required for transferring the concept to acoustics. Hence, we now focus our attention on the acoustic gyrator. We realize such a device by considering a simple acoustic waveguide filled with a fluid on which a velocity of $\nu=\nu_{0} \hat{x}$ is applied. Considering the so-called Doppler effect, ${ }^{14}$ one can easily deduce that the signals transmitting from the left to the right side of such a waveguide will acquire an additional phase shift

(a)

(b)

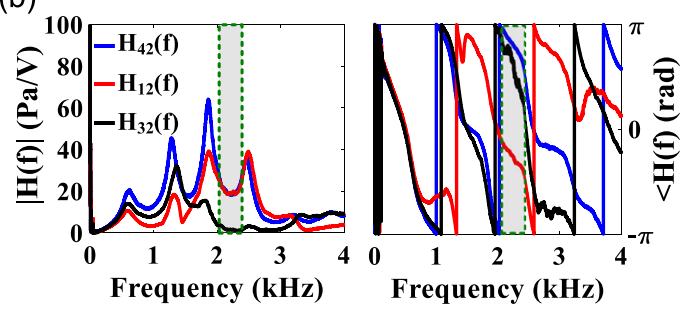

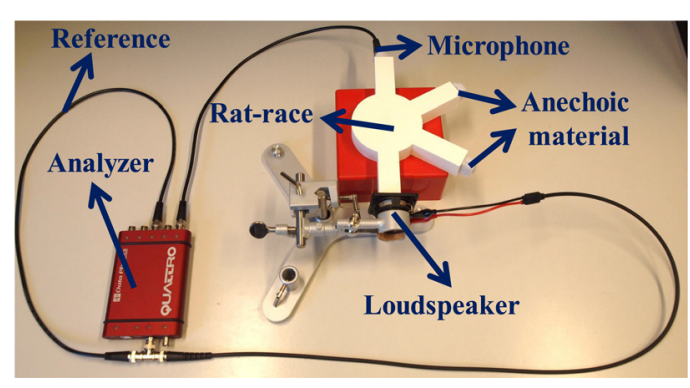

(c)

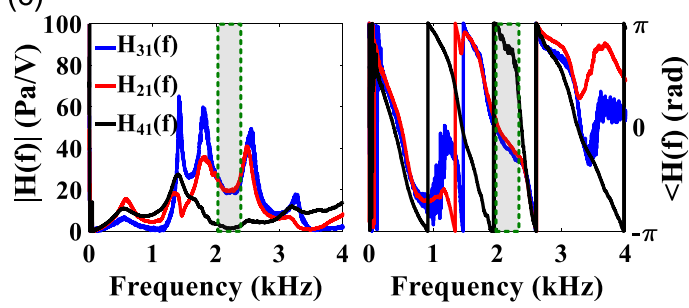

FIG. 3. (Color online) Experimental demonstration of the acoustic rat-race coupler. (a) Experimental setup used to evaluate the functionality of the fabricated rat-race coupler. (b) Magnitude (left) and phase (right) of the corresponding transfer functions when the rat race is excited from port 2. (c) Same as panel (b) except that the excitation is placed at port 1 . The experimental results are consistent with our numerical findings. 
(a)

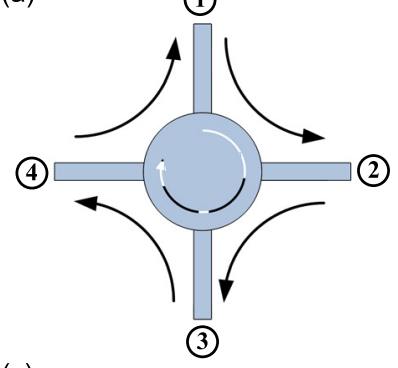

(c)

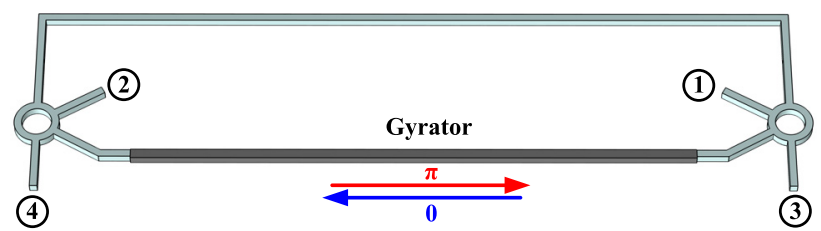

FIG. 4. (Color online) Construction of an acoustic four-port circulator based on the proposed rat-race coupler. (a) Conceptual picture of a four-port circulator. The power fed into each port of the circulator is solely transmitted to the next port in rotation. (b) An acoustic gyrator, a required ingredient to realize four-port circulators, is constructed making use of a simple waveguide filled with a fluid on which a velocity of $\nu_{0}$ is applied. (c) Proposed structure serving as an acoustic four-port circulator. The device is composed of two rat-race couplers whose collinear arms are connected to each other via a gyrator.

of $\psi=4 \pi f_{0} L \nu_{0} / c^{2}$ compared to the signals travelling from right to left. Assuming $\psi=\pi$, such a simple configuration can be treated as an acoustic gyrator with the scattering matrix of ${ }^{15}$

$$
S=e^{j(\pi / 2)}\left[\begin{array}{cc}
0 & 1 \\
-1 & 0
\end{array}\right] .
$$

Now that we have all the required ingredients, we construct the acoustic four-port circulator represented in Fig. 4(a), taking inspiration from its microwave counterpart. ${ }^{11}$ The configuration includes two distinct rat-race couplers whose collinear arms are connected to each other and a gyrator. When sound is fed into port 1 , it splits into two identical signals arriving in phase and with the same amplitude at the collinear arms of the second rat-race coupler. These two signals are then added up to appear at port 2, while their subtraction leads to a zero sound pressure at port 4 . Notice that the port 3 is already isolated from the excitation thanks to the isolation that the first rat-race coupler provides. The sound injected into port 2 will similarly be split into two identical signals by the second rat-race coupler. These two signals, however, arrive out-of-phase at the collinear arms of the first rat-race coupler due to additional $\pi$ phase shift enforced by the gyrator. Consequently, their summation results in a pressure of zero at port 1 , while their subtraction gives rise to a non-zero pressure level at port 3 . By following the same arguments, one can easily find that the injected sound into port 3 is received only at port 4 , and the sound injected into port 4 only emerges at port 1 .

In order to numerically investigate the proposed circulator, we excite it from port 1 and calculate the pressure field distribution over the entire structure [Fig. 5(a)]. As observed, the power is transmitted to port 2 but not ports 3 and 4 . Figures 4(b), 4(c) and 4(d) report the pressure field distribution when the excitation is placed at ports 2,3 , and 4 , respectively. The obtained results confirm that the proposed system is indeed working as a four-port circulator. We note that the applicability of the proposed configuration might be hindered by acoustic losses and sensitivity to back reflection at the sharp turns. Furthermore, considering the fact that the circulator is built from resonating components and works based on interference phenomenon, it does not provide a very large bandwidth of operation and might be sensitive to geometrical tolerances. Its relatively large size could be decreased by considering resonant implementations for the gyrator and coupling waveguides. (a)

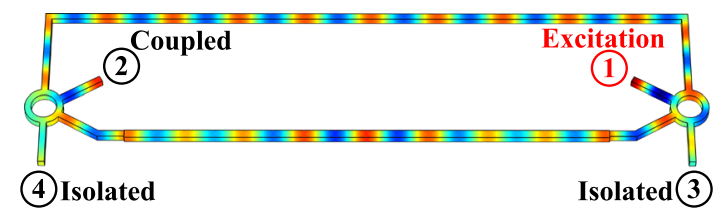

(b)

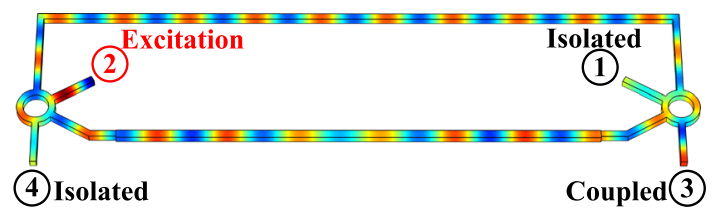

(c)

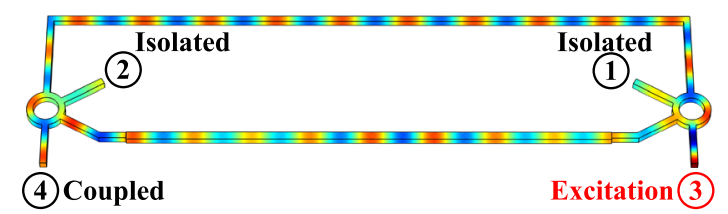

(d)

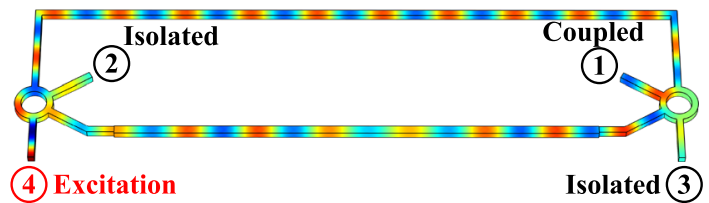

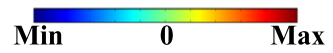

FIG. 5. (Color online) Full-wave demonstration of the proposed four-port circulator. (a) When power is injected into port 1, it splits between the two collinear arms of the first rat-race coupler. These two signals, arriving in phase and with the same amplitude at the collinear arms of the second magic tee, are then added up to appear at port 2 while their subtraction reduces the pressure level to zero at port 4 . (b) When power is injected into port 2 , it splits between the two collinear arms of the second magic tee. These two signals, however, arrive out-of phase at the collinear arms of the first magic tee. Therefore, their summation reduces to zero at port 1 whereas their subtraction results in a non-zero sound pressure level received at port 3 . (c), (d) Pressure profile over the entire structure when ports 3 and 4 are excited, respectively. 


\section{CONCLUSION}

To conclude, we proposed an acoustic rat-race coupler mimicking the scattering behavior of microwave magic-tees. In contrast to magic-T hybrid waveguides, the proper functionality of the proposed rat-race coupler is well understood on the basis of constructive and destructive interferences between the clockwise and counterclockwise modes of a ring resonator. An acoustic four-port circulator was also designed and demonstrated leveraging the proposed acoustic rat-race coupler. Full wave simulations were provided to confirm the proper behavior of the proposed devices. We believe that the peculiar isolation offered by such junctions can open a plethora of new opportunities for transporting a large variety of well-known microwave devices such as duplexers, mixers, power combiners, power dividers, switches, and modulators to acoustics, expanding the toolkit of modern acoustic engineering. ${ }^{16-67}$

\section{ACKNOWLEDGMENTS}

This work was supported by the Swiss National Science Foundation (SNSF) under Grant No. 172487.

(a)

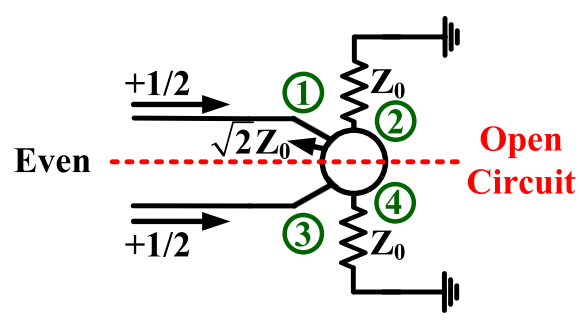

(b)
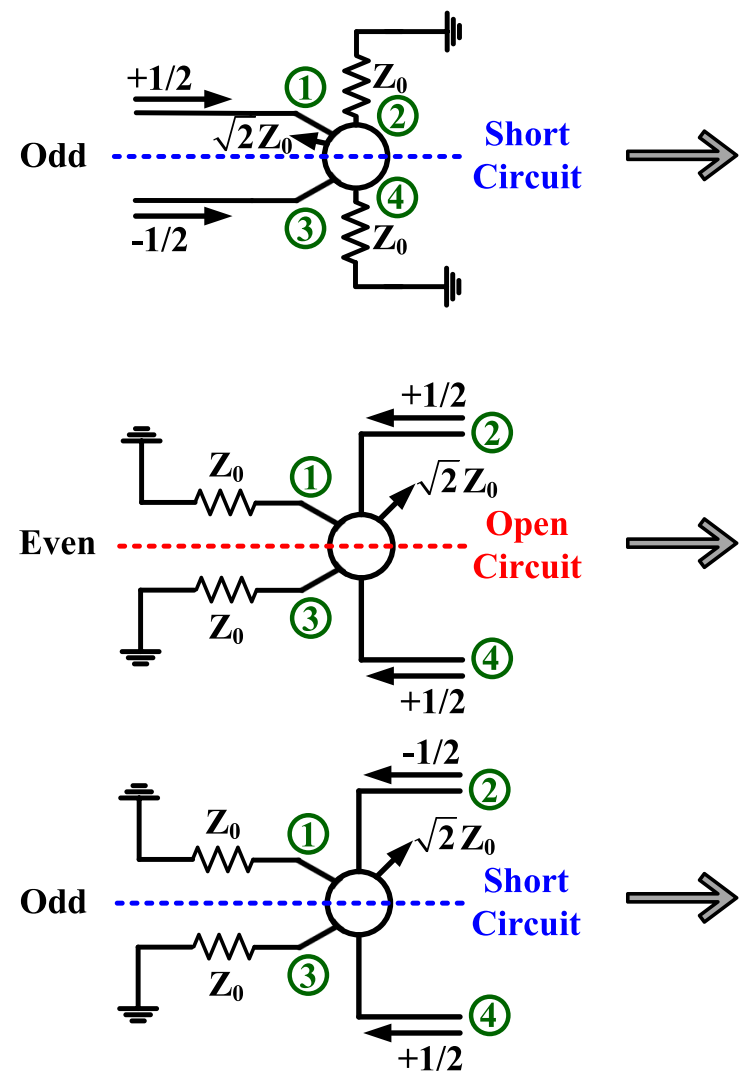

APPENDIX A: EVEN-ODD MODE ANALYSIS

Here, we describe the even-odd mode analysis of the proposed acoustic rat-race coupler. Assume that a sound wave of unit amplitude is injected into port 1 of the rat-race coupler. Suppose further that the characteristic impedance of the ring resonator is $\sqrt{2}$ times that of the arm waveguides. Employing the even-odd mode analysis technique, ${ }^{9}$ we can decompose the configuration into the superposition of two transmission line models and excitations as depicted in Fig. 6(a). One can then readily write the corresponding scattered pressure fields of the form

$$
\begin{aligned}
& S_{11}=\frac{1}{2} R_{e}+\frac{1}{2} R_{o}, \\
& S_{21}=\frac{1}{2} T_{e}+\frac{1}{2} T_{o}, \\
& S_{31}=\frac{1}{2} R_{e}-\frac{1}{2} R_{o}, \\
& S_{41}=\frac{1}{2} T_{e}-\frac{1}{2} T_{o},
\end{aligned}
$$
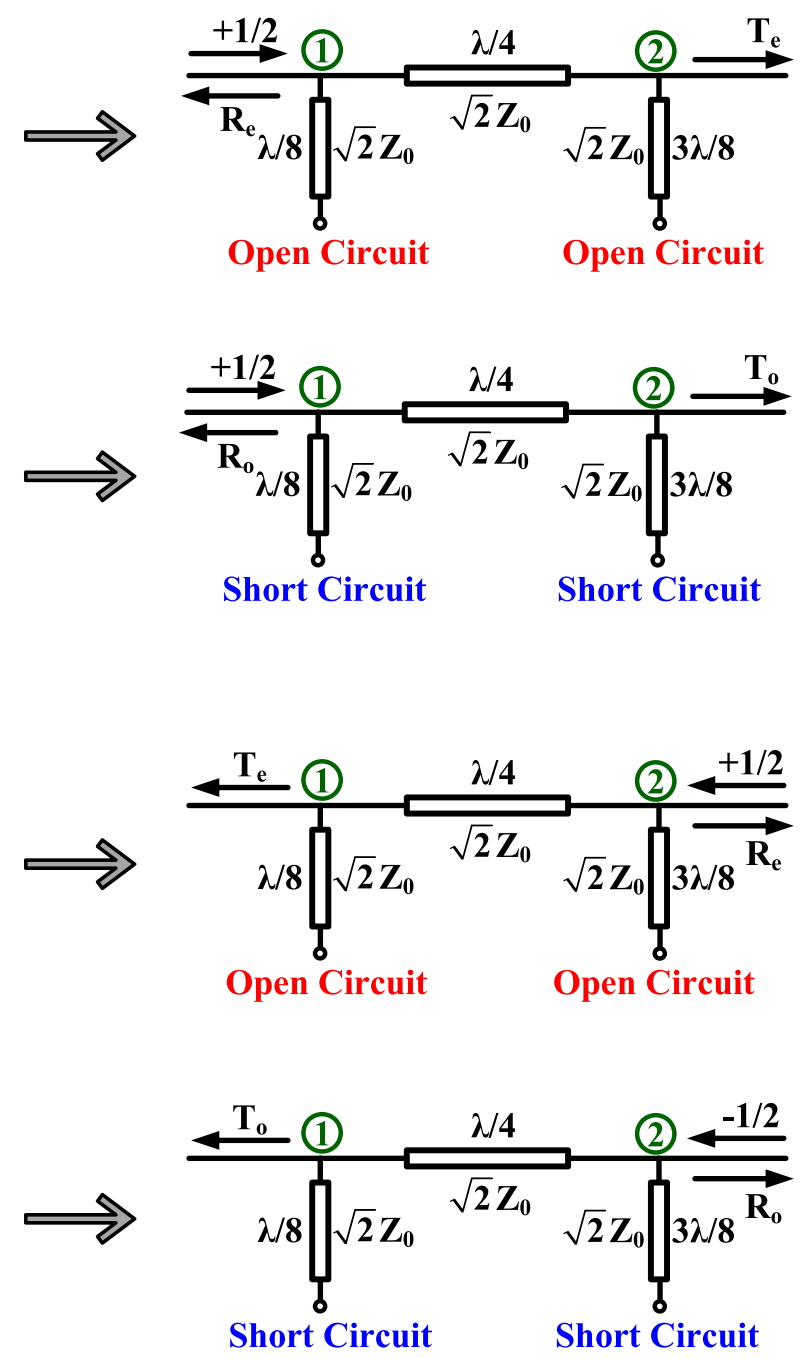

FIG. 6. (Color online) Even-odd mode analysis for the proposed acoustic rat race coupler. (a) Assume sound wave of unit amplitude is injected into port 1 of the rat-race coupler. The corresponding scattering parameters can be obtained by decomposing the problem into the even (top panel) and odd (bottom panel) transmission lines and excitations. (b) Same as panel (a) except that the excitation is assumed to be placed at port 4. 
where $R_{e}\left(T_{e}\right)$, and $R_{o}\left(T_{o}\right)$ are the reflection (transmission) coefficients of the transmission lines associated with the even and odd modes, respectively, which are of the form

$$
\begin{aligned}
& R_{e}=-\frac{j}{\sqrt{2}}, \\
& T_{e}=-\frac{j}{\sqrt{2}}, \\
& R_{o}=\frac{j}{\sqrt{2}}, \\
& T_{o}=-\frac{j}{\sqrt{2}} .
\end{aligned}
$$

Substituting Eq. (A2) into Eq. (A1), one obtains

$$
\begin{aligned}
& S_{11}=0, \\
& S_{21}=-\frac{j}{\sqrt{2}}, \\
& S_{31}=-\frac{j}{\sqrt{2}}, \\
& S_{41}=0,
\end{aligned}
$$

which forms the first row and column of the scattering matrix presented in Eq. (3). Next suppose the rat-race coupler is excited from port 4. Similar to the previous case, one can decompose this case into two simpler transmission lines and excitation as shown in Fig. 6(b), from which the amplitudes of the scattered waves can be obtained as

$$
\begin{aligned}
S_{14} & =\frac{1}{2} T_{e}-\frac{1}{2} T_{o}, \\
S_{24} & =\frac{1}{2} R_{e}-\frac{1}{2} R_{o}, \\
S_{34} & =\frac{1}{2} T_{e}+\frac{1}{2} T_{o}, \\
S_{44} & =\frac{1}{2} R_{e}+\frac{1}{2} R_{o},
\end{aligned}
$$

in which

$$
\begin{aligned}
& R_{e}=\frac{j}{\sqrt{2}}, \\
& T_{e}=-\frac{j}{\sqrt{2}}, \\
& R_{o}=-\frac{j}{\sqrt{2}}, \\
& T_{o}=-\frac{j}{\sqrt{2}} .
\end{aligned}
$$

Substituting Eq. (A5) into Eq. (A4) then results in the following scattering parameters:

$$
\begin{aligned}
& S_{14}=0, \\
& S_{24}=\frac{j}{\sqrt{2}}, \\
& S_{34}=\frac{-j}{\sqrt{2}}, \\
& S_{44}=0,
\end{aligned}
$$

which forms the fourth row and column of the scattering matrix given in Eq. (3). The remaining elements of the scattering matrix can be easily obtained from symmetry arguments.

\section{APPENDIX B: NUMERICAL METHODS}

The simulations throughout the manuscript were performed using Comsol Multiphysics 5.3, acoustic module, solving the acoustic wave equation using finite element method (FEM). Sound hard walls boundary conditions (Neumann boundary condition for the pressure field) were applied to the walls of the rat-race. We obtained the frequency spectrum of the scattering parameters by exciting the rat-race from one port with a plane wave of unit amplitude, and recording the sound pressure levels at other ports.

\section{APPENDIX C: GEOMETRICAL DETAILS}

The rat-race coupler is composed of a ring resonator with the inner radius of $3 \mathrm{~cm}$ and the outer radius of $4.4 \mathrm{~cm}$, which is connected to four square waveguides with the side length of $2 \mathrm{~cm}$. Notice that, in order to achieve perfect matching between the waveguides and the ring, the side length of the waveguide is designed to be $\sqrt{2}$ times the difference between the inner and outer radii of the ring.

${ }^{1}$ H. A. Bethe, "Theory of diffraction by small holes," Phys. Rev. 66, 163-182 (1944).

${ }^{2}$ R. Levy, "Synthesis of symmetrical branch-guide directional couplers," IEEE Trans. Microwave Theory Tech. 16, 995-1006 (1968).

${ }^{3}$ J. Hirokawa, K. Sakurai, M. Ando, and N. Goto, "An analysis of a waveguide T junction with an inductive post," IEEE Trans. Microwave Theory Tech. 39, 563-566 (1991).

${ }^{4}$ E. J. Wilkinson, "An N-way hybrid power divider," IEEE Trans. Microwave Theory Tech. 8, 118 (1960).

${ }^{5}$ W. A. Tyrrell, "Hybrid circuits for microwaves," Proc. IRE 35, 1294-1306 (1947).

${ }^{6}$ M. Park and B. Lee, "A dual-band Wilkinson power divider," IEEE Microwave Wireless Compon. Lett. 18, 85-87 (2008).

${ }^{7}$ Y. Sun and A. Freundorfer, "Broadband folded Wilkinson power combiner/splitter," IEEE Microwave Wireless Compon. Lett. 14, 295-297 (2004).

${ }^{8}$ Y. Cheng, W. Hong, and K. Wu, "Novel substrate integrated waveguide fixed phase shifter for 180-degree directional coupler," in 2007 IEEE/ MTT-S Int. Microwave Symp., pp. 189-192.

${ }^{9}$ D. Pozar, Microwave Engineering, 4th ed. (Wiley, New York, 2005).

${ }^{10}$ R. B. Mouw, "A broad-band hybrid junction and application to the star modulator,” IEEE Trans. Microwave Theory Tech. 16, 911-918 (1968).

${ }^{11}$ A. Das and S. K. Das, Microwave Engineering, 2nd ed. (McGraw-Hill, New Dehli, 2009), p. 465.

${ }^{12}$ H. J. Riblet, "Balanced duplexer," U.S. patent 2,586,993 (February 26, 1952).

${ }^{13}$ F. F. He, K. Wu, W. Hong, L. Han, and X. Chen, "A planar magic-T structure using substrate integrated circuits concept and its mixer applications," IEEE Trans. Microwave Theory Tech. 59, 72-79 (2011).

${ }^{14}$ E. J. Reed, M. Soljačić, and J. D. Joannopoulos, "Reversed Doppler effect in photonic crystals," Phys. Rev. Lett. 91, 133901 (2003).

${ }^{15}$ F. Zangeneh Nejad and R. Fleury, "Doppler-based acoustic gyrator,"Appl. Sci. 8, 1083 (2018).

${ }^{16} \mathrm{H}$. Hashemi and B. Analui, "All-acoustic duplexers using directional couplers," U.S. patent 9,866,201 (January 9, 2018).

${ }^{17}$ M. Shin, F. M. Fazi, P. A. Nelson, and F. C. Hirono, "Mode-matchingbased sound field recording and synthesis with circular double-layer arrays," J. Sound Vib. 333, 3794-3817 (2014).

${ }^{18}$ R. Fleury, D. L. Sounas, C. F. Sieck, M. R. Haberman, and A. Alù, "Sound isolation and giant linear nonreciprocity in a compact acoustic circulator," Science 343, 516-519 (2014). 
${ }^{19}$ R. Fleury, D. Sounas, and A. Alù, “An invisible acoustic sensor based on parity-time symmetry,” Nat. Commun. 6, 5905 (2015).

${ }^{20}$ R. Fleury, D. Sounas, M. R. Haberman, and A. Alù, "Non-reciprocal acoustics," Acoust. Today 11, 14-21 (2015).

${ }^{21}$ S. Bhuktare, A. Bose, H. Singh, and A. A. Tulapurkar, "Gyrator based on magneto-elastic coupling at a ferromagnetic/piezoelectric interface," Sci. Rep. 7, 840 (2017).

${ }^{22}$ A. Blanchard, T. P. Sapsis, and A. F. Vakakis, "Non-reciprocity in nonlinear elastodynamics,” J. Sound Vib. 412, 326-335 (2018).

${ }^{23}$ F. Li, P. Anzel, J. Yang, P. G. Kevrekidis, and C. Daraio, "Granular acoustic switches and logic elements," Nat. Commun. 5, 5311 (2014).

${ }^{24}$ Z. M. Gu, J. Hu, B. Liang, X. Y. Zou, and J. C. Cheng, "Broadband nonreciprocal transmission of sound with invariant frequency," Sci. Rep. 6, 19824 (2016).

${ }^{25}$ C. Fu, B. Wang, T. Zhao, and C. Q. Chen, "High efficiency and broadband acoustic diodes," Appl. Phys. Lett. 112, 051902 (2018).

${ }^{26}$ L. M. Brekhovskikh, Y. P. Lysanov, and J. P. Lysanov, Fundamentals of Ocean Acoustics (Springer Science \& Business Media, New York, 2003).

${ }^{27}$ J. H. Oh, H. W. Kim, P. S. Ma, H. M. Seung, and Y. Y. Kim, "Inverted biprism phononic crystals for one-sided elastic wave transmission applications," Appl. Phys. Lett. 100, 213503 (2012).

${ }^{28}$ Y. Li, B. Liang, Z. M. Gu, X. Y. Zou, and J. C. Cheng, "Unidirectional acoustic transmission through a prism with near-zero refractive index," Appl. Phys. Lett. 103, 053505 (2013).

${ }^{29}$ Y. L. Huang, H. X. Sun, J. P. Xia, S. Q. Yuan, and X. L. Ding, "Multiband asymmetric acoustic transmission in a bended waveguide with multiple mechanisms," Appl. Phys. Lett. 109, 013501 (2016).

${ }^{30}$ M. Maldovan, "Sound and heat revolutions in phononics," Nature 503, 209-217 (2013).

${ }^{31}$ H. Jia, M. Ke, C. Li, C. Qiu, and Z. Liu, "Unidirectional transmission of acoustic waves based on asymmetric excitation of Lamb waves," Appl. Phys. Lett. 102, 153508 (2013).

${ }^{32}$ M. B. Zanjani, A. R. Davoyan, A. M. Mahmoud, N. Engheta, and J. R. Lukes, "One-way phonon isolation in acoustic waveguides," Appl. Phys. Lett. 104, 081905 (2014).

${ }^{33}$ B. I. Popa and S. A. Cummer, "Nonreciprocal active metamaterials," Phys. Rev. B 85, 205101 (2012).

${ }^{34}$ H. X. Sun and S. Y. Zhang, "Enhancement of asymmetric acoustic transmission," Appl. Phys. Lett. 102(11), 113511 (2013).

${ }^{35}$ A. Cicek, O. Adem Kaya, and B. Ulug, "Refraction-type sonic crystal junction diode," Appl. Phys. Lett. 100, 111905 (2012).

${ }^{36}$ M. Xiao, G. Ma, Z. Yang, P. Sheng, Z. Q. Zhang, and C. T. Chan, "Geometric phase and band inversion in periodic acoustic systems," Nat. Phys. 11, 240-244 (2015).

${ }^{37}$ H. Esfahlani, S. Karkar, H. Lissek, and J. R. Mosig, “Acoustic dispersive prism," Sci. Rep. 6, 18911 (2016).

${ }^{38}$ A. A. Maznev, G. Gu, S. Y. Sun, J. Xu, Y. Shen, N. Fang, and S. Y. Zhang, "Extraordinary focusing of sound above a soda can array without time reversal," New J. Phys. 17, 042001 (2015).

${ }^{39}$ J. Xu, X. Jiang, N. Fang, E. Georget, R. Abdeddaim, J. M. Geffrin, M. Farhat, P. Sabouroux, S. Enoch, and S. Guenneau, "Molding acoustic, electromagnetic and water waves with a single cloak," Sci. Rep. 5, 10678 (2015).

${ }^{40}$ X. Fan, C. Qiu, S. Zhang, M. Ke, and Z. Liu, "Highly asymmetric interaction forces induced by acoustic waves in coupled plate structures," J. Appl. Phys. 118, 244506 (2015).

${ }^{41}$ S. Zhang, Y. Zhang, Y. Guo, Y. Leng, W. Feng, and W. Cao, "Realization of subwavelength asymmetric acoustic transmission based on lowfrequency forbidden transmission," Phys. Rev. Appl. 5, 034006 (2016).

${ }^{42}$ K. Song, J. Kim, S. Hur, J. H. Kwak, S. H. Lee, and T. Kim, "Directional reflective surface formed via gradient-impeding acoustic meta-surfaces," Sci. Rep. 6, 32300 (2016)

${ }^{43}$ X. P. Wang, L. Le Wan, T. N. Chen, Q. X. Liang, and A. L. Song, "Broadband acoustic diode by using two structured impedance-matched acoustic metasurfaces,” Appl. Phys. Lett. 109, 044102 (2016).
${ }^{44}$ S. Zhai, H. Chen, C. Ding, F. Shen, C. Luo, and X. Zhao, "Manipulation of transmitted wave front using ultrathin planar acoustic metasurfaces," Appl. Phys. A: Mater. Sci. Process. 120, 1283-1289 (2015).

${ }^{45}$ F. Zangeneh-Nejad and R. Fleury, "Acoustic birefringence via nonEulerian metamaterials," arXiv:1803.09070 (2018).

${ }^{46}$ T. Devaux, V. Tournat, O. Richoux, and V. Pagneux, "Asymmetric acoustic propagation of wave packets via the self-demodulation effect," Phys. Rev. Lett. 115, 234301 (2015).

${ }^{47}$ Y. F. Zhu, X. Y. Zou, B. Liang, and J. C. Cheng, “Acoustic one-way open tunnel by using metasurface,” Appl. Phys. Lett. 107, 113501 (2015).

${ }^{48}$ F. Zangeneh-Nejad and R. Fleury, "Acoustic analogues of high-index optical waveguide devices,” Sci. Rep. 8, 10401 (2018).

${ }^{49}$ R. Fleury, A. B. Khanikaev, and A. Alù, "Floquet topological insulators for sound," Nat. Commun. 7, 11744 (2016).

${ }^{50}$ Z. Shen, Y. L. Zhang, Y. Chen, C. L. Zou, Y. F. Xiao, X. B. Zou, F. W. Sun, G. C. Guo, and C. H. Dong, "Experimental realization of optomechanically induced non-reciprocity," Nat. Photon. 10, 657-661 (2016).

${ }^{51}$ C. He, X. Ni, H. Ge, X. C. Sun, Y. Bin Chen, M. H. Lu, X. P. Liu, and Y. F. Chen, "Acoustic topological insulator and robust one-way sound transport," Nat. Phys. 12, 1124-1129 (2016).

${ }^{52} \mathrm{G}$. Trainiti and M. Ruzzene, "Non-reciprocal elastic wave propagation in spatiotemporal periodic structures,” New J. Phys. 18, 083047 (2016).

${ }^{53}$ C. Liu, Z. Du, Z. Sun, H. Gao, and X. Guo, "Frequency-preserved acoustic diode model with high forward-power-transmission rate," Phys. Rev. Appl. 3, 064014 (2015).

${ }^{54}$ R. Sasaki, Y. Nii, Y. Iguch, and Y. Onose, "Nonreciprocal propagation of surface acoustic wave in Ni/LiNbO 3," Phys. Rev. B 95, 020407 (2017).

${ }^{55} \mathrm{H}$. Zhu and M. Rais-Zadeh, "Non-reciprocal acoustic transmission in a GaN delay line using the acoustoelectric effect," IEEE Electron Dev. Lett. 38, 802-805 (2017).

${ }^{56}$ R. Fleury, D. Sounas, and A. Alu, "Non-reciprocal acoustic devices based on spatio-temporal angular-momentum modulation," J. Acoust. Soc. Am. 136, 2281 (2014).

${ }^{57}$ Y. G. Peng, C. Z. Qin, D. G. Zhao, Y. X. Shen, X. Y. Xu, M. Bao, H. Jia, and X. F. Zhu, "Experimental demonstration of anomalous Floquet topological insulator for sound," Nat. Commun. 7, 13368 (2016).

${ }^{58}$ A. V. Poshakinskiy and A. N. Poddubny, "Phonoritonic crystals with a synthetic magnetic field for an acoustic diode," Phys. Rev. Lett. 118, 156801 (2017).

${ }^{59}$ F. Zangeneh-Nejad and R. Fleury, "Performing mathematical operations using high-index acoustic metamaterials," New J. Phys. 20, 073001 (2018).

${ }^{60}$ H. Abbaszadeh, A. Souslov, J. Paulose, H. Schomerus, and V. Vitelli, "Sonic Landau levels and synthetic gauge fields in mechanical metamaterials," Phys. Rev. Lett. 119, 195502 (2017).

${ }^{61}$ B. Rostami-Dogolsara, M. K. Moravvej-Farshi, and F. Nazari, "Tunable phononic structures using Lamb waves in a piezoceramic plate," Phys. Rev. B 99, 094302 (2016).

${ }^{62}$ X. Yang, J. Yin, G. Yu, L. Peng, and N. Wang, “Acoustic superlens using Helmholtz-resonator-based metamaterials," Appl. Phys. Lett. 107, 193505 (2015).

${ }^{63}$ X. Jiang, B. Liang, X. Y. Zou, J. Yang, L. L. Yin, J. Yang, and J. C. Cheng, "Acoustic one-way metasurfaces: Asymmetric phase modulation of sound by subwavelength layer," Sci. Rep. 6, 28023 (2016).

${ }^{64}$ Q. Wang, Y. Yang, X. Ni, Y. L. Xu, X. C. Sun, Z. G. Chen, L. Feng, X. P. Liu, M. H. Lu, and Y. F. Chen, "Acoustic asymmetric transmission based on time-dependent dynamical scattering," Sci. Rep. 5, 10880 (2015).

${ }^{65}$ F. Zangeneh-Nejad and R. Fleury, "Topological fano resonances," Phys. Rev. Lett 122, 014301 (2019).

${ }^{66}$ B. Liang, X. S. Guo, J. Tu, D. Zhang, and J. C. Cheng, "An acoustic rectifier," Nat. Mater. 9, 989-992 (2010).

${ }^{67}$ Y. Li, B. Liang, Z. M. Gu, X. Y. Zou, and J. C. Cheng, "Reflected wavefront manipulation based on ultrathin planar acoustic metasurfaces," Sci. Rep. 3, 2546 (2013). 